\title{
Ultrafast Self-Assembly Using Ultrasound: A Facile Route to the Rapid Fabrication of Well-Ordered Dense Arrays of Inorganic Nanostructures**
}

\author{
Anitha Ethirajan,* Sathya Punniyakoti, Marc D'Olieslaeger, Patrick Wagner, and \\ Hans-Gerd Boyen
}

The use of self-assembly for generating ordered ensembles of functional nanostructures at surfaces has seen significant progress in the last decade owing to the simplicity and lowcost of the process. In particular, block copolymers (BCP) comprising chemically distinct blocks that can phase-separate into nanoscaled domains owing to mutual incompatibility between the blocks have extensively been exploited for selfassembly. ${ }^{[1-4]}$ In case of poly(styrene)-block-poly(vinyl pyridine) (PS- $b$-PVP), by using an apolar solvent such as toluene, the BCP self-assembles into spherical reverse micelles with PVP and PS forming the core and the corona, respectively. When such micelles are loaded with inorganic precursor salts and then transferred onto substrates by dip coating or spin coating, they spontaneously arrange themselves into 2D arrays. ${ }^{[5]}$ By subsequent plasma treatments, inorganic nanodot arrays can be achieved thereby preserving the original arrangement of the micellar ensembles. ${ }^{[6-8]}$ The flexibility to control both the particle size and the interparticle spacing has enabled intensive studies in different fields including optics, ${ }^{[9,10]}$ optoelectronics, ${ }^{[11]}$ magnetic recording media, ${ }^{[12]}$ catalysis, ${ }^{[13,14]}$ chemical reactivity, ${ }^{[15,16]}$ nanotubes and nanowires, ${ }^{[17-19]}$ and biomedicine. ${ }^{[20-22]}$ Furthermore, micellar ensembles were successfully utilized as masks to nanopattern surfaces. ${ }^{[1,5,11,23,24]}$

Although micelles are widely used as templates for the creation of various nanomaterials, their formation as equilibrium structures and their homogeneous loading with metal salts (Scheme 1a) still remains a challenging task: ${ }^{[25]}$ stirring of micellar solutions on extended timescales ranging from several days up to weeks or even months (in case of transition metals) are required ${ }^{[25]}$ depending on the degree of solubility of the precursor salt in the solvent and the efficiency of binding of the metal ions to the micellar nanoreactors.

[*] Dr. A. Ethirajan ${ }_{,}^{[+]}$S. Punniyakoti, ${ }^{[+]}$Prof. Dr. M. D'Olieslaeger, Prof. Dr. P. Wagner, Prof. Dr. H.-G. Boyen

Institute for Materials Research (IMO), Hasselt University

Wetenschapspark 1, 3590 Diepenbeek (Belgium)

E-mail: anitha.ethirajan@uhasselt.be

Prof. Dr. M. D'Olieslaeger, Prof. Dr. P. Wagner, Prof. Dr. H.-G. Boyen IMEC, division IMOMEC

3590 Diepenbeek (Belgium)

$\left[{ }^{+}\right]$These authors contributed equally to this work.

[**] Financial support by the Research Foundation Flanders (FWO) within the Odysseus program, the projects G.0456.12, G.0346.09N and the Methusalem project "NANO" is gratefully acknowledged. We furthermore thank J. Baccus and C. Willems for technical support. A.E. is a postdoctoral research fellow of the FWO.
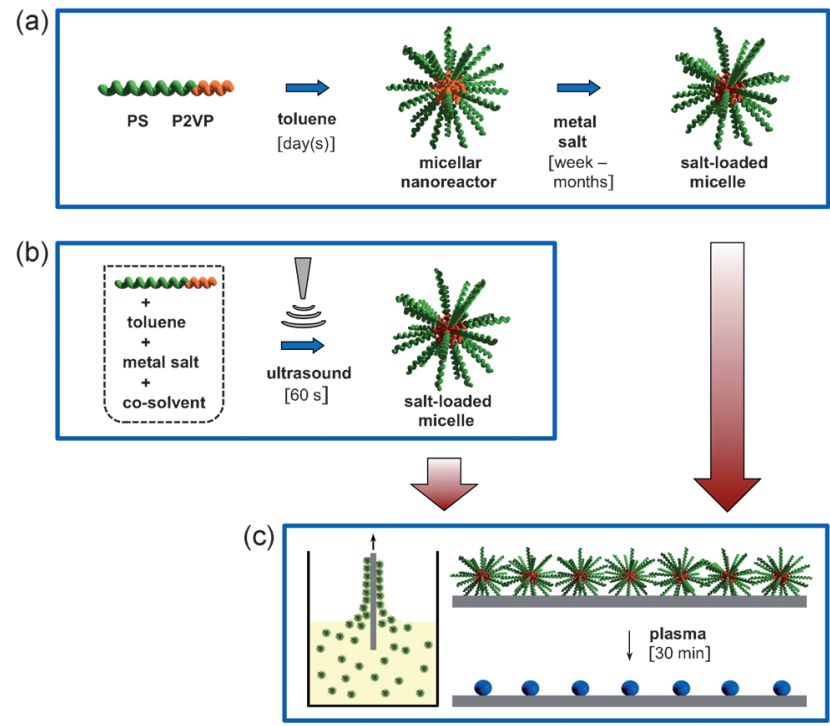

Scheme 1. The preparation of metal-salt-loaded micelles by conventional stirring (a) or by the new route based on ultrasonication in the presence of a co-solvent (b). After depositing micellar monolayers on top of appropriate substrates by dip-coating, ordered arrays of metal oxide or metal nanoparticles are obtained by applying oxygen/hydrogen plasmas (c).

Therefore, the development of new methods to speed up the preparation is crucial but demanding as well, as the array quality achievable with existing methods should not be compromised by a new synthesis route. Herein, we report a simple and convenient approach for the rapid, one-step preparation of precursor salt-loaded micelles that can be transferred onto a substrate with a high lateral order without the need of additional pre- or post-treatments. ${ }^{[26-29]}$ As illustrated in Scheme $1 \mathrm{~b}$, this rapid fabrication is achieved by mixing the BCP, the solvent and the precursor salt together with a small amount of an additional co-solvent followed by ultrasonication for a very short time $(60 \mathrm{~s})$. After transfer onto a substrate and subsequent removal of the organic constituents by plasma treatments, a highly ordered dense array of inorganic nanoparticles is achieved (Scheme 1c).

High shear forces induced by sound waves have extensively been utilized in heterophase systems, especially in case of miniemulsions, ${ }^{[30]}$ thereby facilitating the synthesis of rather complex polymer particles. ${ }^{[31]}$ By applying ultrasound, the disruption of already formed micelles could be expected which, consequently, would result in a broad distribution of micellar fragments and thus a poor quality of the final 
nanoparticle array. However, recent work demonstrates that the energy provided by sound waves is able to disrupt larger aggregates of micelles rather than to break individual micelles into smaller fragments. ${ }^{[32]}$ Thus, the presence of non-agglomerated, intact micelles during sonication could be anticipated which should favor their homogeneous loading with precursor salt. As sound waves should furthermore increase the diffusion of metal ions within the solution, the loading of micelles with precursor salt is expected to happen significantly faster as compared to conventional stirring. This diffusion could even be more accelerated by simultaneously adding an appropriate co-solvent that is miscible in the continuous phase and that is able to dissolve the precursor salt.

It is worth mentioning that, on one hand, co-solvents in micellar solutions have already demonstrated their great potential for fine-tuning the morphology of micellar assemblies. ${ }^{[33,34]}$ On the other hand, post-treatment by ultrasound annealing has been found to improve the degree of order within micellar ensembles as well. ${ }^{[35]}$ However, to the best of our knowledge, no efforts have been made so far to combine ultrasonication and co-solvents to synthesize salt-loaded micellar structures on short timescales, which is the aim of the present work.

In our experiment, PS- $b$-P2VP, toluene, a co-solvent (methanol, ethanol, water), and a precursor salt $\left(\mathrm{FeCl}_{3}\right.$, $\mathrm{TiCl}_{3}, \mathrm{HAuCl}_{4}$ ) are all added together into a glass vial and immediately subjected to ultrasound using a sonicating horn for a very short time $(60 \mathrm{~s})$. The impact of ultrasonication with/without co-solvent on the formation of micelles loaded with $\mathrm{FeCl}_{3}$ can be seen in Figure 1. AFM results (left side) and corresponding autocorrelation functions (right side) are presented, measured after transferring micellar monolayers onto silicon substrates by dip-coating. Focusing first on results obtained by conventionally stirring a micellar solution for one week (Figure 1a), a rather poor degree of lateral ordering can be recognized in the autocorrelation arising from largely fluctuating diameters of individual micelles. When adding a small amount of methanol as a co-solvent $(50 \mu \mathrm{L}$ as compared to $5 \mathrm{~mL}$ toluene) and stirring again for one week, a slightly better result is obtained as indicated by the presence of short-range order in the autocorrelation function (Figure $1 \mathrm{~b}$ ). This situation is rather comparable to using ultrasonication for $60 \mathrm{~s}$ without any co-solvent (Figure 1c). However, the latter result is achieved on the basis of a reduction in processing time by 4 orders of magnitude ( 60 s versus 1 week). Finally, if a small amount of methanol is present during sonication $(50 \mu \mathrm{L}$ as compared to $5 \mathrm{~mL}$ toluene), a significantly improved lateral order is obtained demonstrating now the development of medium-range order (Figure 1d). Thus, when combining ultrasonication with an appropriate cosolvent, a significantly improved micellar pattern can be created on a very short timescale thus leaving the conventional preparation route falling far behind our new approach.

While significant progress in the synthesis of uniform micelles can be inferred from Figure 1, their homogeneous loading with precursor salt, and consequently the uniform size of the resulting inorganic nanoparticles, remains to be demonstrated. Simultaneously, the impact of different co-
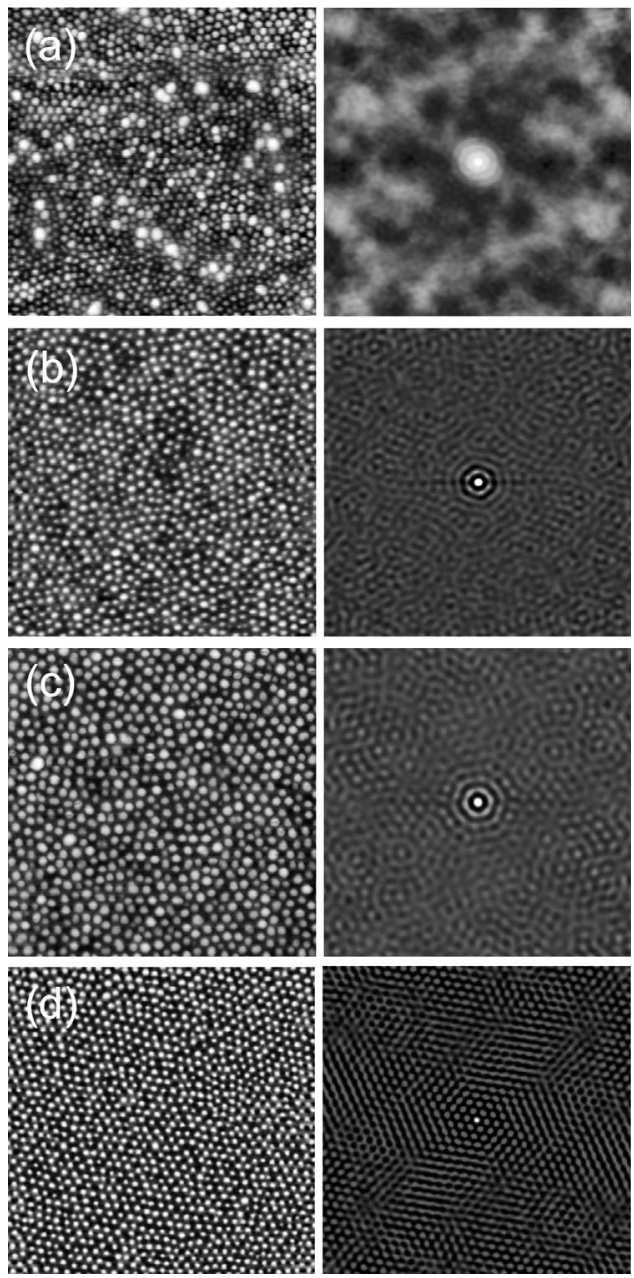

Figure 1. AFM results (left column) and related auto-covariance (right column) of iron-salt-loaded micellar monolayers derived from solutions prepared by a) conventional stirring for 1 week in the absence of any co-solvent; b) conventional stirring for 1 week in the presence of methanol; c) sonication for $60 \mathrm{~s}$ without additional co-solvent; and d) ultrasonication for $60 \mathrm{~s}$ in the presence of methanol (all images $1 \times 1 \mu m^{2}$ ).

solvents needs to be studied as well to permit optimization of the new procedure. Therefore, AFM measurements were carried out after exposure of appropriate micellar arrays to an oxygen plasma, which allows all organic parts to be removed. ${ }^{[7]}$ Figure 2 presents AFM images (left side) and related autocorrelation functions (right side) of iron oxide nanoparticle ensembles resulting from either one week of conventional stirring (Figure $2 \mathrm{a}$ ) or $60 \mathrm{~s}$ ultrasonication in the presence of ethanol, methanol, as well as water. The latter is a co-solvent that does not show a high solubility within the continuous phase. Using these AFM images, size distributions of the final nanoparticles can be deduced. Starting with the conventional stirring approach, strongly fluctuating particle sizes can be inferred from Figure $2 \mathrm{a}(3.0 \pm 0.8 \mathrm{~nm})$ accompanied by a rather poor lateral order. When switching to the new approach with ethanol as co-solvent (Figure 2b), the distribution in particle size appears to be improved (2.8 \pm $0.6 \mathrm{~nm}$ ) while the array is still lacking a high degree of hexagonal order. However, if methanol is used as co-solvent 


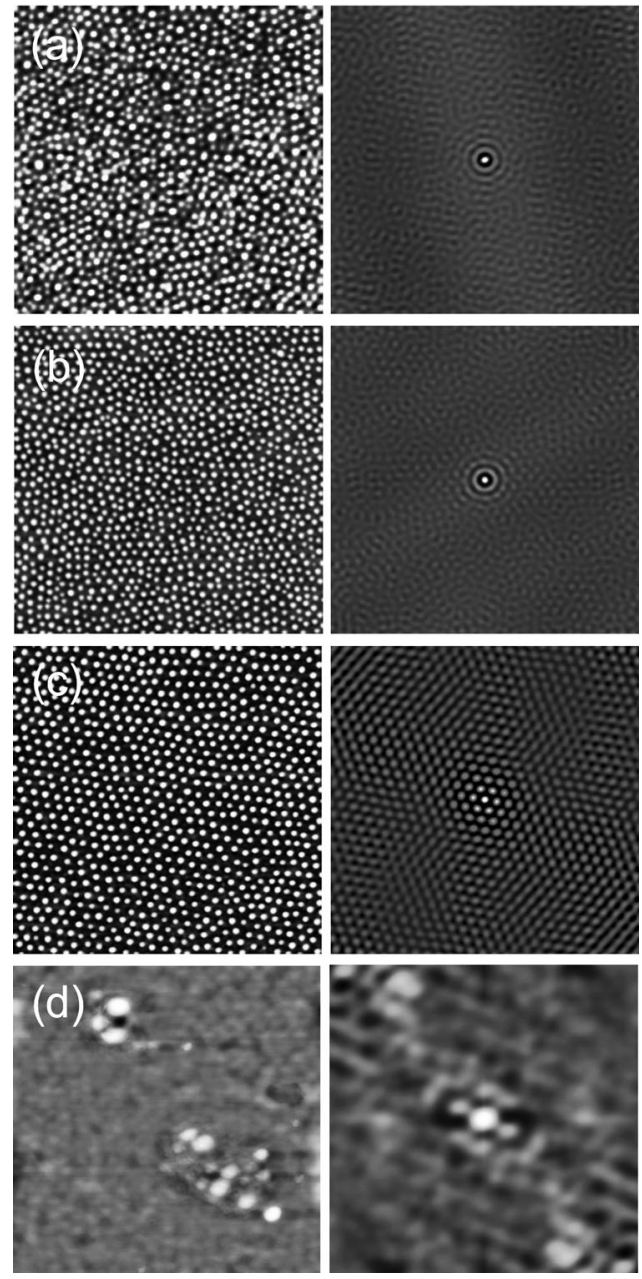

Figure 2. AFM images (left column) and related auto-covariance (right column) of iron oxide nanoparticle arrays derived from solutions prepared by a) conventional stirring for 1 week in the absence of any co-solvent; b) $60 \mathrm{~s}$ ultrasound in the presence of ethanol; c) $60 \mathrm{~s}$ ultrasound in the presence of methanol; and d) $60 \mathrm{~s}$ ultrasound in the presence of water (all images $1 \times 1 \mu \mathrm{m}^{2}$ ). From these AFM images, the distribution in particle size can be extracted: $3.0 \pm 0.8 \mathrm{~nm}$ (a); $2.8 \pm 0.6 \mathrm{~nm}$ (b); and $3.0 \pm 0.3 \mathrm{~nm}$ (c).

(Figure 2c), an excellent lateral order is achieved accompanied by a significantly improved size distribution (3.0 \pm $0.3 \mathrm{~nm}$ ). In clear contrast, the use of water as co-solvent ends up in nanoparticle agglomerates suffering from large fluctuations in particle size and the complete loss of lateral order. Thus, applying high shear forces in the presence of methanol enables to deposit even transition metal-based nanoparticle arrays with significantly improved lateral order on an unprecedented short timescale.

As the time required to reach equilibrium during the formation of salt-loaded micelles certainly depends on the nature of the metal salt itself, the new method was also tested for auric acid $\left(\mathrm{HAuCl}_{4}\right)$. While transition-metal salts might bind to the P2VP blocks in a more complex way, ${ }^{[36]}$ in case of auric acid, the $\mathrm{AuCl}^{4-}$ ions bind to the pyridine units, forming a pyridinium salt. ${ }^{[6]}$ Figure 3 presents gold salt-loaded micelles prepared by ultrasonication $(60 \mathrm{~s})$ in the presence of methanol. As compared to gold-salt loaded micelles synthesized
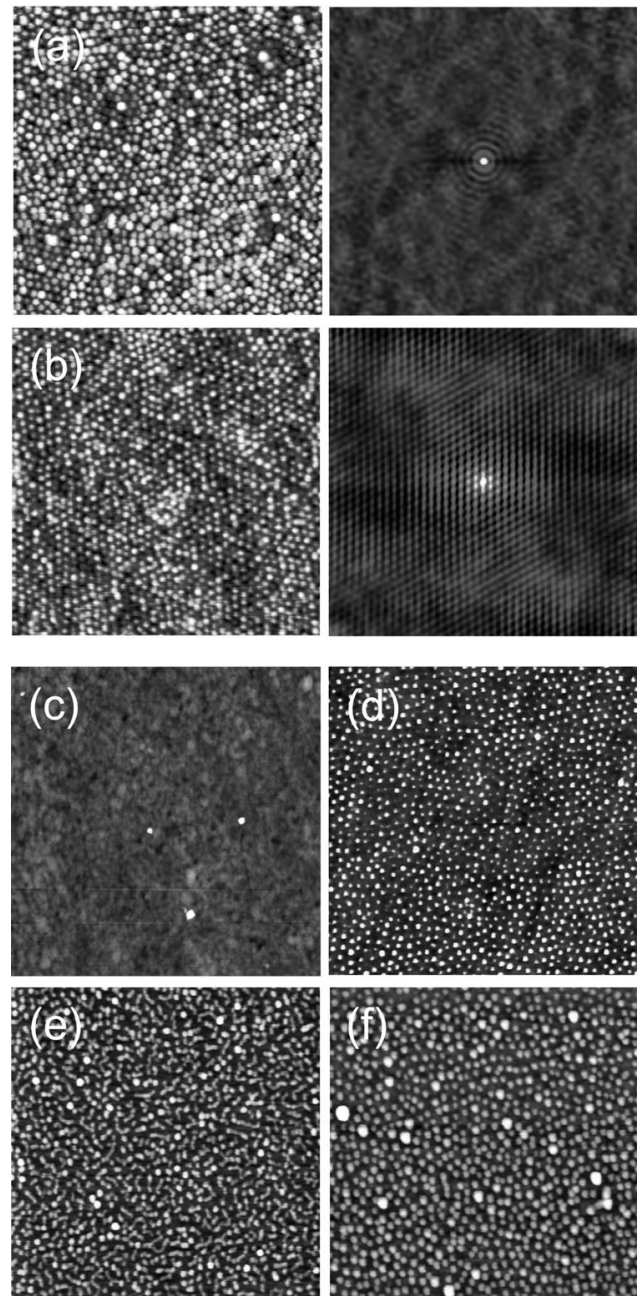

Figure 3. AFM results (left column) and related auto-covariance (right column) of gold-salt-loaded micellar monolayers derived from different solutions: conventional stirring for 1 week (a) and after $60 \mathrm{~s}$ ultrasound in the presence of methanol (b). AFM also reveals that the loading of $\mathrm{TiCl}_{3}$ into micellar cores using conventional stirring for 1 week is completely unsuccessful as evidenced by the absence of nanoparticles after plasma treatment (c). In contrast, the new approach allows to load micelles within $60 \mathrm{~s}$ (d). Raising the loading ratio (ratio between metal ions and pyridine units) from 0.3 (d) to

1 (e), thereby increasing the particle size from $1.9 \pm 0.6 \mathrm{~nm}$ to $3.3 \pm 1.2 \mathrm{~nm}$, results in a significant fraction of worm-like structures for $\mathrm{TiCl}_{3}$ (e) and a loss of hexagonal order for $\mathrm{FeCl}_{3}(\mathrm{f})$, for which a size of $4.1 \pm 1.4 \mathrm{~nm}$ is obtained (all images $1 \times 1 \mu \mathrm{m}^{2}$ ).

via conventional stirring ( 1 week, Figure 3 a), the use of the new approach clearly results in a striking improvement of the lateral order (Figure $3 \mathrm{~b}$ ) accompanied by a reduction of the synthesis time by 4 orders of magnitude.

The influence of sonication time as well as co-solvent concentration was also studied. Briefly, on one hand, increasing the timescale of sonication to several minutes did not affect the quality of the final array, thus pointing to an equilibrium of the micellar solutions which is already reached within the first minute. On the other hand, sonication times beyond $4 \mathrm{~min}$ lead to excessive evaporation of the (co-)solvent owing to an overall temperature increase. Furthermore, raising the amount of methanol ( $100 \mu \mathrm{L}$ and above $)$ did 
not improve the lateral order of the final array indicating that $50 \mu \mathrm{L}$ was an optimum amount. It is important to note that, as all the experiments were performed using a Ti-based sonication horn, contaminations by Ti species could be expected for the final nanoparticle arrays. However, such contaminations could be ruled out by means of a careful chemical analysis using XPS.

Preliminary studies demonstrated that other transition metal-based salts like $\mathrm{MoCl}_{5}, \mathrm{NbCl}_{5}$, or $\mathrm{TiCl}_{3}$ can efficiently be transferred into micellar nanoreactors in the same way, thus indicating a rather general applicability of our new technique. This is especially important in the latter case, where conventional stirring does not allow even small amounts of $\mathrm{TiCl}_{3}$ to be loaded into the P2VP cores within reasonable timescales. This is evidenced by Figure $3 \mathrm{c}$, which by the absence of any nanoparticle array after plasma treatment, reveals the existence of completely empty micelles after 1 week stirring. In contrast, our technique allows such loading within $60 \mathrm{~s}$ (Figure $3 \mathrm{~d}$ ).

Owing to the observed efficiency of the loading process combining ultrasound and an appropriate co-solvent, loading ratios (that is, the ratio between metal ions and pyridine units) beyond the value of 0.5 as found for the conventional approach $^{[6-7]}$ could be anticipated. However, in case of $\mathrm{TiCl}_{3}$, increasing the loading ratio to unity induces the formation of a significant fraction of worm-like structures, besides well-separated nanoparticles (Figure $3 \mathrm{e}$ ). In case of $\mathrm{FeCl}_{3}$ (Figure $3 \mathrm{f}$ ), such structures are absent. However, the array order is clearly reduced here leaving a value of 0.5 as a loading limit similar to that found for the conventional approach.

To this end, the fundamental question arises why the timescale to form and load micellar structures with precursor materials can be accelerated by many orders of magnitude allowing even materials such as $\mathrm{TiCl}_{3}$ to be loaded, which do not diffuse into the micellar cores under normal stirring conditions. During the course of chemical reactions (in this case, self-assembly of BCP micelles plus simultaneous formation of organometallic complexes between pyridine units and metal ions), reactant molecules need to surmount an energy barrier to allow their transformation into products. The energy needed for this process is usually provided by heat, light, or pressure, which often excites reactants to higher energy states, thereby facilitating passing the energy barrier $^{[37]}$ A fundamentally different way of initiating or accelerating chemical reactions is the use of mechanical forces as provided by ultrasound (sonochemistry ${ }^{[38]}$ ). These forces expose reacting molecules to stress fields, which allow rearrangement reactions to be accelerated or even new reaction pathways to be favored to yield products not obtainable from purely thermal or light-induced reactions. ${ }^{[37]}$

In general, sonochemistry can be divided into 2 regimes: 1) a low-frequency range $(10-100 \mathrm{kHz})$ where the resulting wavelength is of the order of $\mathrm{cm}$ (in our case $20 \mathrm{kHz}$, which translates into a wavelength of about $6 \mathrm{~cm}$ in toluene at $300 \mathrm{~K}$ ); and 2) a high-frequency range up to $10 \mathrm{GHz}$ where the wavelength approaches the $100 \mathrm{~nm}$ scale, that is, the typical size of macromolecular structures. While the first type of sound waves are usually defocused owing to the large wavelength, the latter allows strongly focused acoustic beams providing enough energy to even break weak chemical bonds (as used for example in controlled drug-delivery applications). ${ }^{[39]}$ In the low-frequency range, sound waves are not energetic enough to break chemical bonds. Rather, the energy causes the formation, growth and implosive collapse of bubbles in the liquid (cavitation) ${ }^{[38]}$ During collapse, intense heating of the bubbles occurs, which form local hot spots with temperatures reaching $5000^{\circ} \mathrm{C}$, pressures of $500 \mathrm{bar}$, and lifetimes of few $\mu$ s in an otherwise cold liquid. ${ }^{[38]}$ Consequently, on one hand, for micelles in the vicinity of such hot spots, significantly enhanced diffusion of the metal salt through the polymeric shell can be anticipated during the short lifetime of the rapid thermal annealing process. On the other hand, the shock waves emitted during the collapse of the cavities might produce local stress fields that are strong enough to activate the formation of stable complexes between metal ions and pyridine units along chemical pathways that are not accessible under normal stirring conditions, as can be inferred from the case of $\mathrm{TiCl}_{3}$. Of course, this simplified picture cannot explain the need of a small amount of cosolvent required to achieve the final results. Thus, to develop a more elaborate picture about the underlying sonochemistry, support from theory would be desirable.

It is worth to point out that, after $60 \mathrm{~s}$ sonication, the micellar solutions must be close to equilibrium as indicated by their homogeneity: all of the solutions prepared this way did not require any filtration before their deposition on substrates (to remove larger aggregates), in contrast to conventional stirring. The presence of homogeneous solutions can also be inferred from the observed improved hexagonal order and the small size distribution of the resulting nanoparticle arrays. Homogeneous micellar solutions can be expected even in case of inhomogeneous energy density profiles within the glass vial during sonication, as the energy density should affect the rate of cavitation but not the energy released upon the collapse of individual cavities. It is this latter energy, however, which very likely controls the dynamics of micelle formation/salt loading in the vicinity of an imploding cavity.

In conclusion, a new approach is demonstrated that allows the fabrication of metal-salt-loaded micelles on an unprecedented short timescale. This facile route serves as an efficient platform for the subsequent deposition of well-ordered nanoparticle arrays at surfaces. The method is based on the rapid formation and salt-loading of micellar nanoreactors by combining both ultrasonication and the addition of an appropriate co-solvent. This way, even transition-metalbased nanoparticle arrays can be deposited on timescales that are accelerated by several orders of magnitude as compared to the more conventional routes. This rapid synthesis might boost future studies in a broad range of research areas where high-quality nanoparticle arrays are required with high size-selectivity and high degree of lateral order. Furthermore, this approach might open the way to the functionalization of surfaces by nanomaterials even at an industrial scale owing to its simplicity, effectiveness, and low cost, especially regarding fabrication time. 


\section{Experimental Section}

Diblock copolymers (PS[27700]- $b$-P2VP[4300], PS[32500]-b-P2VP[7800]) were purchased from Polymer Source Inc. (Canada). Metal salts $\left(\mathrm{HAuCl}_{4}, \mathrm{FeCl}_{3}, \mathrm{TiCl}_{3}\right)$, dried toluene (max. $0.001 \% \mathrm{H}_{2} \mathrm{O}$ ), ethanol (p.a), and methanol (p.a) were purchased from Sigma Aldrich. The salts were dissolved in $50 \mu \mathrm{L}$ co-solvent and mixed with $25 \mathrm{mg}$ of the BCP together with $5 \mathrm{~mL}$ of toluene. The resulting mixture was immediately sonicated by means of a Branson 450 Digital Sonifier $(20 \mathrm{kHz}, 400 \mathrm{~W})$ employing a $1 / 8^{\prime \prime}$ (ca. $3 \mathrm{~mm}$ ) Ti tip. The optimized procedure used $65 \%$ amplitude for $60 \mathrm{~s}$ (2 pulses of $30 \mathrm{~s}$ separated by a $20 \mathrm{~s}$ pause) to avoid excessive overall heating. Additionally, all samples were cooled using an ice bath during sonication. In the absence of co-solvent, the salt and the BCP were added to the toluene prior to sonication. In case of conventional stirring, the polymer was stirred in toluene for 3 days prior to the salt loading (with/without co-solvent) followed by an additional week of stirring.

The micelles were transferred to Si substrates by dip-coating $\left(6 \mathrm{~mm} \mathrm{~min}^{-1}\right)$. The organic layer was removed by oxygen plasma ( $10 \mathrm{~W}, 30 \mathrm{~min}, 0.02 \mathrm{mbar}$ ) using a custom-built plasma etcher. The etched samples were characterized in-situ by XPS using a PHI 5600 LS electron spectrometer equipped with monochromatized Xrays $(1486.6 \mathrm{eV})$. The topography of the resulting arrays was studied by a Nanoscope IIIa multimode AFM (Digital Instruments, Veeco) using the tapping mode. Particle analysis including the calculation of the autocorrelation function was performed using Nanoscope software version 5 .

Received: March 8, 2013

Revised: June 9, 2013

Published online: July 24, 2013

Keywords: block copolymers - nanoparticles - self-assembly . ultrasound

[1] M. Park, C. Harrison, P. M. Chaikin, R. A. Register, D. H Adamson, Science 1997, 276, 1401.

[2] S. Förster, M. Antonietti, Adv. Mater. 1998, 10, 195.

[3] J. Bang, U. Jeong, D. Y. Ryu, T. P. Russell, C. J. Hawker, $A d v$. Mater. 2009, 21, 4769.

[4] H.-C. Kim, S.-M. Park, W. D. Hinsberg, Chem. Rev. 2010, 110, 146.

[5] J. P. Spatz, T. Herzog, S. Mössmer, P. Ziemann, M. Möller, $A d v$. Mater. 1999, 11, 149 .

[6] J. P. Spatz, S. Mössmer, C. Hartmann, M. Möller, T. Herzog, M. Krieger, H. G. Boyen, P. Ziemann, B. Kabius, Langmuir 2000, 16, 407.

[7] G. Kästle, H. G. Boyen, F. Weigl, G. Lengl, T. Herzog, P. Ziemann, S. Riethmüller, O. Mayer, C. Hartmann, J. P. Spatz, M Möller, M. Ozawa, F. Banhart, M. G. Garnier, P. Oelhafen, $A d v$. Funct. Mater. 2003, 13, 853.

[8] R. E. Palmer, S. Pratontep, H. G. Boyen, Nat. Mater. 2003, 2, 443.

[9] A. M. Popa, B. Wenger, E. Scolan, G. Voirin, H. Heinzelmann, R. Pugin, Appl. Surf. Sci. 2009, 256, S12.

[10] S. Gilb, K. Hartl, A. Kartouzian, J. Peter, U. Heiz, H. G. Boyen, P. Ziemann, Eur. Phys. J. D 2007, 45, 501.

[11] M. Haupt, S. Miller, A. Ladenburger, R. Sauer, K. Thonke, J. P. Spatz, S. Riethmüller, M. Möller, F. Banhart, J. Appl. Phys. 2002 91, 6057.
[12] A. Ethirajan, U. Wiedwald, H. G. Boyen, B. Kern, L. Y. Han, A. Klimmer, F. Weigl, G. Kästle, P. Ziemann, K. Fauth, J. Cai, R. J. Behm, A. Romanyuk, P. Oelhafen, P. Walther, J. Biskupek, U. Kaiser, Adv. Mater. 2007, 19, 406.

[13] B. R. Cuenya, S. H. Baeck, T. F. Jaramillo, E. W. McFarland, J. Am. Chem. Soc. 2003, 125, 12928.

[14] L. K. Ono, B. Roldan-Cuenya, Catal. Lett. 2007, 113, 86.

[15] H. G. Boyen, G. Kästle, F. Weigl, B. Koslowski, C. Dietrich, P. Ziemann, J. P. Spatz, S. Riethmüller, C. Hartmann, M. Möller, G. Schmid, M. G. Garnier, P. Oelhafen, Science 2002, 297, 1533.

[16] H. G. Boyen, A. Ethirajan, G. Kästle, F. Weigl, P. Ziemann, G. Schmid, M. G. Garnier, M. Büttner, P. Oelhafen, Phys. Rev. Lett. 2005, 94, 016804.

[17] M. Haupt, A. Ladenburger, R. Sauer, K. Thonke, R. Glass, W. Roos, J. P. Spatz, H. Rauscher, S. Riethmüller, M. Möller, J. Appl. Phys. 2003, 93, 6252.

[18] C. Hinderling, Y. Keles, T. Stöckli, H. F. Knapp, T. de Los Arcos, P. Oelhafen, I. Korczagin, M. A. Hempenius, G. J. Vancso, R. Pugin, H. Heinzelmann, Adv. Mater. 2004, 16, 876.

[19] B. N. Mbenkum, A. S. Schneider, G. Schütz, C. Xu, G. Richter, P. A. van Aken, G. Majer, J. P. Spatz, ACS Nano 2010, 4, 1805.

[20] J. H. Huang, S. V. Grater, F. Corbellinl, S. Rinck, E. Bock, R. Kemkemer, H. Kessler, J. D. Ding, J. P. Spatz, Nano Lett. 2009, 9, 1111.

[21] B. Geiger, J. P. Spatz, A. D. Bershadsky, Nat. Rev. Mol. Cell Biol. 2009, 10, 21

[22] A. C. Pearson, E. Pound, A. T. Woolley, M. R. Linford, J. N. Harb, R. C. Davis, Nano Lett. 2011, 11, 1981.

[23] M. Haupt, S. Miller, R. Glass, M. Arnold, R. Sauer, K. Thonke, M. Möller, J. P. Spatz, Adv. Mater. 2003, 15, 829 .

[24] F. Weigl, S. Fricker, H. G. Boyen, C. Dietrich, B. Koslowski, A. Plettl, O. Pursche, P. Ziemann, P. Walther, C. Hartmann, M. Ott, M. Möller, Diamond Relat. Mater. 2006, 15, 1689.

[25] J. Polleux, M. Rasp, I. Louban, N. Plath, A. Feldhoff, J. P. Spatz, Acs Nano 2011, 5, 6355.

[26] J. Y. Cheng, A. M. Mayes, C. A. Ross, Nat. Mater. 2004, 3, 823.

[27] R. A. Segalman, A. Hexemer, R. C. Hayward, E. J. Kramer, Macromolecules 2003, 36, 3272.

[28] G. Kim, M. Libera, Macromolecules 1998, 31, 2569.

[29] D. E. Angelescu, J. H. Waller, R. A. Register, P. M. Chaikin, Adv. Mater. 2005, 17, 1878.

[30] K. Landfester, Angew. Chem. 2009, 121, 4556; Angew. Chem. Int. Ed. 2009, 48, 4488.

[31] K. Schöller, A. Ethirajan, A. Zeller, K. Landfester, Macromol. Chem. Phys. 2011, 212, 1165

[32] J. P. Zhao, S. Pispas, G. Z. Zhang, Macromol. Chem. Phys. 2009, 210, 1026.

[33] H. W. Shen, A. Eisenberg, Angew. Chem. 2000, 112, 3448; Angew. Chem. Int. Ed. 2000, 39, 3310.

[34] T. Pietsch, N. Gindy, A. Fahmi, Polymer 2008, 49, 914.

[35] C. Williges, W. W. Chen, C. Morhard, J. P. Spatz, R. Brunner, Langmuir 2013, 29, 989.

[36] A. Riskin, A. M. Beale, H.-G. Boyen, A. Vantomme, A. Hardy, M. K. Van Bael, Phys. Chem. Chem. Phys. 2013, 15, 1675.

[37] C. R. Hickenboth, J. S. Moore, S. R. White, N. R. Sottos, J. Baudry, S. R. Wilson, Nature 2007, 446, 423.

[38] K. S. Suslick, Science 1990, 247, 1439.

[39] J. Xuan, O. Boissiere, Y. Zhao, B. Yan, L. Tremblay, S. Lacelle, H. S. Xia, Y. Zhao, Langmuir 2012, 28, 16463. 